\title{
Testing ADAS end-of-line - Avoid the hazardous effects
}

\author{
Frank Heidemann, SET GmbH
}

This manuscript is not available according to publishing restriction.

Thank you for your understanding. 